Florea A., Meinel C. (2021) Successful Knowledge Transfer - A Boost for Regional Innovation. In: Camarinha-Matos L.M., Boucher X., Afsarmanesh H. (eds) Smart and Sustainable Collaborative Networks 4.0. PRO-VE 2021. IFIP Advances in Information and Communication Technology, vol 629. Springer, Cham. https://doi.org/10.1007/978-3-030-85969-5_50

\title{
Successful Knowledge Transfer - A Boost for Regional Innovation
}

\author{
Adrian Florea ${ }^{1}$ and Christoph Meinel $^{2}$ \\ ${ }^{1}$ Computer Science and Electrical Engineering Department, Lucian Blaga University of \\ Sibiu (ULBS), 550025 Sibiu, Romania \\ adrian.florea@ulbsibiu.ro \\ ${ }^{2}$ Digital Engineering Faculty, University of Potsdam/Hasso Plattner Institute (HPI), \\ 14482 Potsdam, Germany \\ christoph.meinel@hpi.de
}

\begin{abstract}
Innovation is the only way the EU can maintain a strong, sustainable and competitive economy. However, at present there are large imbalances between degrees of innovation of EU countries. Statistics of 2018 and 2019 classify Romania as the least innovative country of EU. One solution to mitigate this drawback consists of development of collaborative networks that replicate the best models of innovation from advanced countries and adapt them to underdeveloped countries from Europe. Such a collaborative network was proposed by HPI Potsdam in 2018 to ULBS with the aim to create an active knowledge transfer center between academia and business/society in the Sibiu region. This paper describes how the center contributes to increasing competitiveness in the Sibiu region by creating collaborative networks, digital education and training platforms as well as fostering applied research projects building on HPI's experience in Potsdam, Germany.
\end{abstract}

Keywords: Innovation, Knowledge Transfer, Digital Education Platform

\section{Introduction}

Statistics at European level from 2018 and 2019 show that Romania remains the EU's least innovative country having approx. $31 \%$ of the EU average [1]. The country registered $15 \%$ diminish of the innovation index compared to 2010 . The limited vision of leaders, limited communication and comparison with what is happening only at the local level (within the organization/region/country) and the lack of collaborative activities at the international level, the lack of investments in human resources and scientific research show their long-term effects. For a long time, the gross domestic product (GDP) allocated to education was less than 5\%. According to Eurostat in 2019, Romania has the second lowest public expenditure on education among the 27 EU member states with $3.6 \%$ of GDP [2].

Figure 1 illustrates the four categories of innovative countries from the EU emphasizing the dishonorable place occupied by Romania. The lack of qualified human resources, lack of research investments in both public sector and private companies, lack of any kind of innovation inside small and medium enterprises 
(SME), difficulties to attract and retaining talents in research centers and universities represent the main problems of innovation in Romania (Table 1).

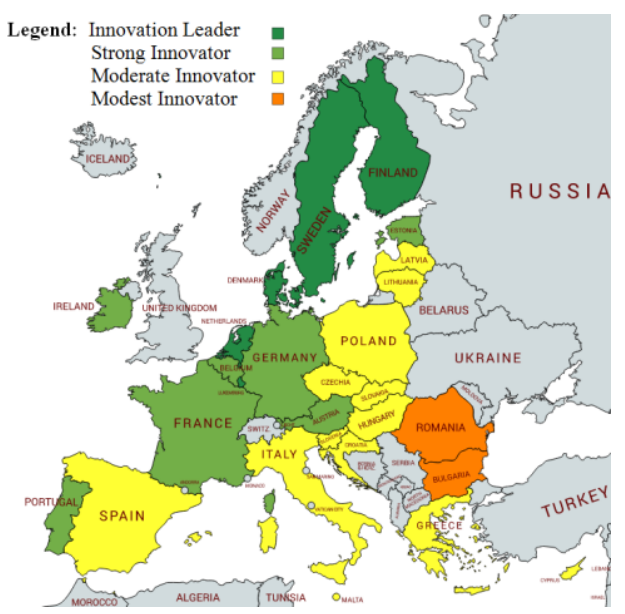

Fig. 1. The 2019 EU map of innovation ${ }^{1}$

Table 1. The main problems of innovation in Romania.

\begin{tabular}{l|c}
\hline Innovation features & Relative to EU in 2019 [\%] \\
\hline SME's Product/Process/Organizational innovations & 0 \\
Lifelong learning & 0 \\
Population with tertiary education & 9.1 \\
Investments in research and development of public sector & 2.9 \\
Enterprises providing ICT training & 5.6 \\
\hline
\end{tabular}

After the European summit from 8-9 May 2019 organized at Sibiu, Romania, the participants came with the recommendation and commitment that Europe can shape its future through research and innovation focusing on cutting-edge research and innovation projects materialized in viable projects being able to become a successful global competitor with the other developed economies of the world [3].

The scientific contribution of this paper consists in proposing of an innovative mechanism of Collaborative Network for territorial innovation. First, the characteristics of competitiveness in the Sibiu region are briefly described. Then, the paper arguments how the creation of collaboration networks, digital education and training platforms, industrial clusters and the use of recent concepts in product development like Design Thinking (DT) and Design Value Creation Process can be considered innovation guidelines in Romania.

Additionally, the paper focuses on the following specific objectives:

01: To prove and emphasize the collaboration for innovation between two institutions (in this case laying the foundations of a Knowledge Transfer Center

\footnotetext{
${ }^{1}$ Source: elaborated by authors using https://mapchart.net/europe.html and data from [1]
} 
(KTC) in Romania at ULBS), showing the sustainable impacts and highlighting the importance of a KTC for accelerated innovation in Factories of the Future (FoF).

O2: To illustrate the knowledge transfer (i) among universities and industrial companies, but also (ii) among different levels of research and innovation groups within HPI to ULBS, through the re-use of German practices that could be applied in Romania. We illustrate also the steps developed for KTC gaining visibility.

\section{Fostering Innovation and Knowledge Transfer: State of the Art}

\subsection{Mission of KTC}

H. Etzkowitz and L. Leydesdorff used first the concept of "technology transfer center" or "knowledge transfer center" in the 1990s [4], by introducing the "Triple Helix" innovation model. Thus, to intensify the interaction between the academic environment (high education institutions - HEIs), industry and government, which favors economic and social development by stimulating innovation, technology transfer centers and industrial parks were proposed. The main role of technology transfer center consists in capitalizing on basic research generated by universities by transferring it to the development of new products, commercial goods, the efficiency of technological processes from industrial companies, following the rules established by, or stimulated by, government through smart specialization strategies, the government also having the role of regulating commercial markets. Thus, the Japanese, well known for aiming the "critical technologies" and knowing that these represent the prerequisites for future industrial growth to a high level, invest in developing their academic research and graduate training capacities [4]. HEIs must confront the transformation from a knowledge-importing economy to a knowledgegenerating economy [5]. Furthermore, the new paradigm and methodology for Digital Innovation is called Open Innovation 2.0 [6]. Its core feature is represented by the "Quadruple Helix" innovation model where government, industry, academia and civil participants work together in a synergistic and faster way to create structural changes.

\subsection{Examples/Case Studies for Knowledge Transfer}

According to [7] "University-industry knowledge transfer is an important source of wealth creation for all partners; however, the practical management of this activity within universities is often hampered".

Hasso Plattner Institute in Potsdam, Germany, was founded in 1998 with the vision to establish a university excellence center in Germany for mastering digital transformation. The approach for making this vision reality was - and still is - to combine excellence in research and teaching with providing an ecosystem for 
innovation while focusing on human needs and the user when developing complex IT systems and software solutions. This combination is the source of value creation at HPI and provides various touch points where knowledge is transferred from the university to external organizations such as enterprises, public institutions or NGO's. The process starts with the design of the study programs which are offered at HPI. All programs include a practice-oriented and research-focused project, called bachelor project and master project or lab, which takes around one to one and a half semester and which is structured as team assignment with the aim to find solutions for real problems posed by external project partners. One example of a successful bachelor's project is the "In-Memory Data Management" project together with SAP in 2008/092. This project resulted in the development of SAP's successful HANA platform. SAP and HPI received the German Innovation Award in 2012 for this joint development.

In many cases, project partners stay in cooperation with the institute in various roles - be it as research partner of HPI's research groups, partner for student projects at the HPI School of Design Thinking ${ }^{3}$, executive education customer of HPI Academy ${ }^{4}$ or as a speaker and supporter of HPI's conferences. This strong network based on the aim to organize effective knowledge transfer as well as to contribute to the economic development in the region and the country has also led to a diversification of HPI's financial resources (Table 2).

Table 2. Third-party funds of HPI in 2020.

\begin{tabular}{l|c}
\hline Financial resources & {$[\%]$} \\
\hline Hasso Plattner Foundation & 59 \\
Public Organizations & 28 \\
Private Organizations & 13 \\
\hline
\end{tabular}

HPI School of Design Thinking started teaching the innovation method in 2007 as the first in Europe. Since then, DT courses are an integral part of HPI's degree programs. HPI School of Design Thinking identified three core elements of design thinking: multidisciplinary team-based mindset, variable creative workspace and iterative process focused on six phases like understanding, observing, formulate of viewpoint, ideate, prototyping and testing.

The teaching offers are complemented by research on design thinking and innovation which contributes to understanding why and how design thinking works in organizations (improves work culture, makes efficient innovation process, reduces the costs) [8]. Additionally, our research highlighted the importance of prototyping in problem-solving and innovation processes [9]. This insight comes into play again in research projects with third parties and is a powerful tool of knowledge transfer.

HPI invests significant energy in projects on digital learning for diverse user groups. One of these projects is openSAP ${ }^{5}$ - a Massive Open Online Courses (MOOC) platform jointly developed together with SAP with the aim to provide online courses for an audience interested in SAP's solutions. The digital education platform,

\footnotetext{
${ }^{2}$ https://hpi.de/plattner/projects/project-archive/bachelor-project-hana.html

3 https://hpi.de/school-of-design-thinking.html

4 https://hpi-academy.de

5 https://open.sap.com/
} 
developed 2011/2012 at HPI, is also used at the World Health Organization ${ }^{6}$ which must ensure that frontline health responders have access to lifesaving knowledge anytime and anywhere. This platform saw a tremendous rise in course enrollments with the spread of COVID-19, as did other HPI's online learning platforms (Fig. 2).

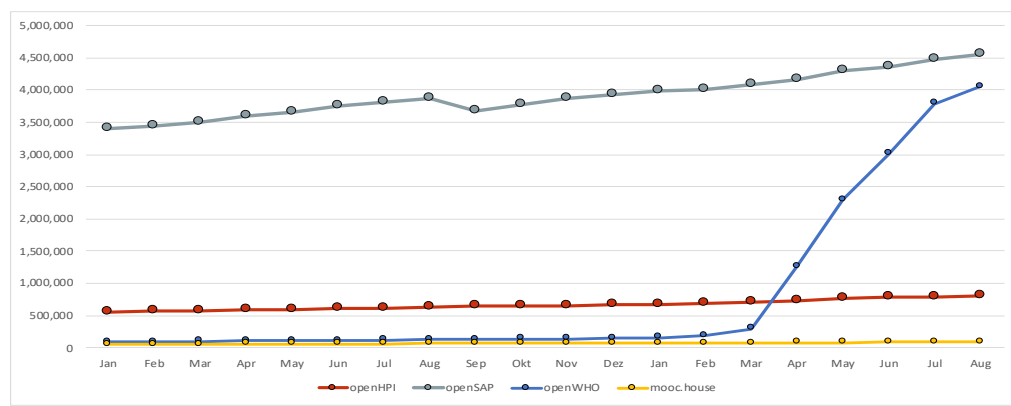

Fig. 2. Course enrollments on openHPI, openSAP, openWHO and mooc.house between Jan. 2019 and Aug. 2020 [10]

While MOOC platforms are designed for a large number of users, HPI also supports knowledge transfer on a smaller scale and targeted toward SMEs. In 2016, the German Federal Ministry for Economic Affairs and Energy started a project funding called 'Gemeinsam digital' (Together digital) to support digitalization in German SMEs. For the federal states, Berlin and Brandenburg, HPI became the project partner. Since then has conducted many workshops on topics like digital business models, how to use AI technologies within a company or how to benefit from digital technologies in HR recruiting. The workshop coaches are connected to HPI chairs so that the current research is transferred to German businesses instantly.

Another novel approach to support innovation in Germany is the 'Digital Hub Initiative ${ }^{7}$ also started by the German Federal Ministry for Economic Affairs and Energy. This initiative supports the establishment of focused innovation hubs in Germany. Currently, there are twelve hubs selected, among them being the 'MediaTech Hub Potsdam' leveraging the historically strong film and media industry in Potsdam, Brandenburg. As part of the hub, the University of Potsdam, the Film University and HPI decided to fund a joint accelerator ${ }^{8}$ to support early-stage startup teams in Potsdam in a 6-month structured program. The accelerator complements the universities' own activities to support students interested in founding a company. At HPI, entrepreneurship is supported by the HPI School of Entrepreneurship ${ }^{9}$ and the Chair of IT-Entrepreneurship established in 2019. They offer courses, competitions, bootcamp, coaching, mentoring as well as research and support students but also alumni and staff on the path of founding and building up their company. The result of this unique infrastructure has contributed in part to the fact that more than 100 startups have now emerged from the HPI's environment. One of them, Signavio, has

\footnotetext{
${ }^{6}$ https://openwho.org/

${ }^{7}$ https://www.de-hub.de/en/

8 https://www.mth-potsdam.de/en/accelerator-en/

9 https://hpi.de/entrepreneurship/hpi-e.html
} 
been bought by SAP for one billion Euros ${ }^{10}$. Although this may be unusual even for the Berlin startup ecosystem, this acquisition of a company initiated through a student bachelor's project by a multinational enterprise shows the importance of early-on investment in entrepreneurship activities at universities, which may contribute significantly to the regional ecosystem boosting innovation.

\section{Collaborative Network for Territorial Innovation}

The collaborative network between HPI and ULBS is a long-term strategic network [11] with two main actions: creating virtual community targeting excellence in research and education and strengthening the collaboration between academia, business and administration sectors, providing virtual laboratory networks and digital collaboration platforms [12] for academic and industrial use to equip students and employees with skills required by Factories of the Future.

In 2018, understanding the need to create an innovation ecosystem that becomes sustainable, HPI has proposed and financially supported ULBS to create a knowledge transfer center, and, in 2020, supported the development of a Digitalization Hub around KTC HPI-ULBS oriented to build capacities through training, coaching and mentoring as well as digital education to maintain and increase the competitiveness of the Sibiu region. Building strong networks and helping regional stakeholders to benefit from the use of digital technologies fosters innovations and new business models attract talents and contribute toward a responsible society with informed citizens. The HPI support targets:

- Access to organizations and experts in Germany and Europe to support SME's to tackle digital technology challenges

- Access to IT capacities, e.g. HPI's Future SOC (Service-Oriented Computing) Lab

- Knowledge transfer and expertise regarding the promotion of entrepreneurship (e.g., accelerator/incubator/startup mentoring)

- Potential cooperation in research projects in the fields of security engineering, knowledge engineering and smart city applications

In Sibiu area the industrial sector predominates. The dynamics of the changes generated by digitalization are first found in industrial companies. At the level of 2017, economic statistics show that the turnover of all companies in the industrial sector in Sibiu County was 3.93 Billion EUR (up from previous years) out of a total of 7.3 Billion EUR (53.84\%). At the country level, Industry was in 2018 the most important economic branch in Romania, with a contribution to the gross added value of $26 \%$, Romania being ranked 4th among the EU countries where the industry fundamentally contributes to the Gross Domestic Product. KTC HPI-ULBS's mission is to contribute to the enhancement of economic competitiveness by stimulating and harnessing the scientific and innovative potential and by assimilating the technological progress in the key areas of ULBS. The objectives are focused on digitalization, innovation, diversity of research teams, cross-fertilization of ideas, and

${ }^{10} \mathrm{https}$ ://www.signavio.com/news/sap-acquisition-signavio/ 
digital transformation with impact on improving the quality of life, economic prosperity, increasing the quality of education and research in Romania.

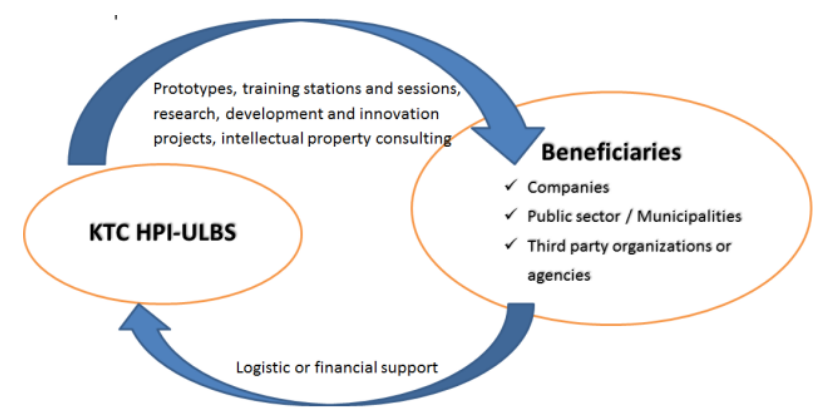

Fig. 3. KTC HPI-ULBS value creation process

KTC HPI-ULBS has as main objective the development and promotion of scientific research, know-how transfer, innovative entrepreneurship and building research, development and innovation infrastructures as well as the promotion of scientific and cultural values for consolidating a knowledge-based society. KTC must exploit the economic potential existing in the Sibiu region and the research skills from ULBS.

The overall outputs (key performance indicators) of KTC HPI-ULBS are:

- Information services (e.g. participation to events), Networking activities

- Training activities, innovation labs and hackathons

- Research contracts with companies / Public-Private Partnerships

- Patent applications and awards

\section{Experimentation and Validation Feedback}

KTC HPI-ULBS has implemented in 2019, thanks to HPI Potsdam and, based on collaboration between OMiLAB NPO, ULBS, industrial cluster PrelMet Transylvania and Continental Sibiu company within DigiFoF ${ }^{11}$ ERASMUS+ KA2 project, a research infrastructure ${ }^{12}$ (Fig. 4) as key asset of a digital ecosystem specific to scientific-innovative activities [13]. Multidisciplinary working teams formed by researchers, students, masters and doctoral students from ULBS but also from partner companies collaborate for developing innovative products / services, acquiring new skills like Creation of Business Ecosystems, Smart Modeling and Digital Twin Engineering. Through the created infrastructure we have:

- enhanced the industry-academia cooperation fostering knowledge transfer and educating about the benefit of digital technologies in the Factories of the Future

- realized vocational training programs using problem-based learning approach and Design Thinking methods (over $90 \mathrm{MSc}$ students and employees from companies)

${ }^{11} \mathrm{https://digifof.eu/}$

12 https://www.omilab.org/nodes/omilab nodes/ulbs/ 
- developed innovative teaching and training materials for students and professionals (6 learning modules, 2 software tools and 4 webinars)

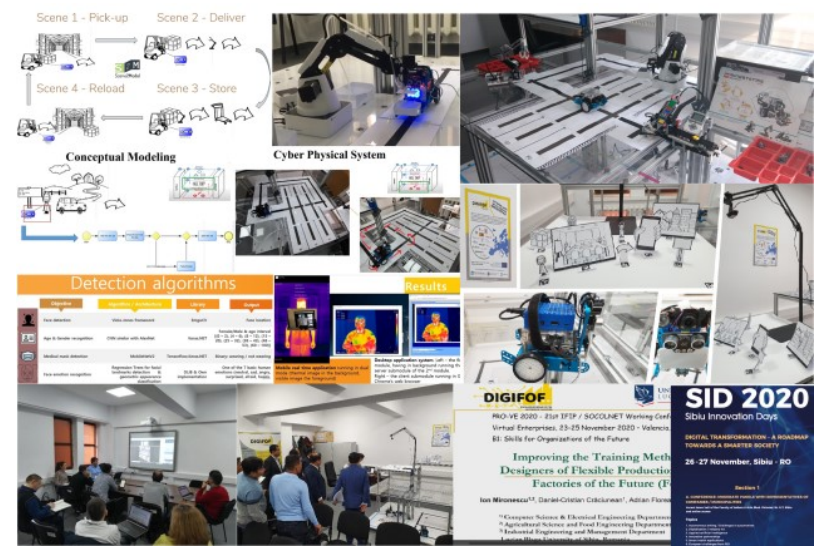

Fig. 4. KTC HPI-ULBS training and research infrastructure

The digital educational platform and experimental environment serve both to research and training activities related to modelling and simulation methods and tools, digitalization and optimization of business process, AI, image processing, collaborative cyber-physical systems and Internet of Things, robotics and automation, smart city, etc. One of the goals is to provide students and professionals the digital skills required by the Factories of the Future in the context of digitalization and Industry 4.0 society [14]. Considering challenges introduced by COVID-19 pandemic, a solution to mitigate its impact was to redefine training ecosystem proposing online training and changing the ULBS teachers' mindset to use and prepare own MOOCs, developing webinars, thereby enlarging the target audience.

The main success factors of the Romanian-German collaboration were the implementation of the KTC within ULBS, the understanding of the potential activities for territorial innovation performed by it, the transfer of knowledge from the research teams from HPI to ULBS and HPI support for developing a favourable mindset for the innovation process in the Sibiu region. Table 3 illustrates some of the key performance indicators achieved by KTC HPI-ULBS in the period 2019-2020.

Of the lessons learned from the collaboration between HPI and ULBS, at least two are worth highlighting (i) digital transformation and value creation through innovation are prerequisites for sustainable development and, (ii) setting international collaborative networks and the adoption of good practices from advanced countries regarding knowledge transfer can be solutions for boosting the territorial innovation.

We cannot talk about barriers in the collaboration between HPI and ULBS; rather, we can highlight obstacles in the implementation of knowledge transfer to Romanian companies, due to legislative specificity and sometimes, the mentality unwilling to change. It is a reserved attitude of Romanian companies in the use of technology transfer services because of their novelty, the difficulty or cost of changing technology or assembly line, or even from lack of trust in universities and research centers. This fact, also observed after the discussions with companies, is in accordance with certain market studies [15], namely, the degree of use of technology 
transfer services $(4.03 \%)$ and product development services $(6.71 \%)$ is very small, and can be a basis for developing this type of service in Sibiu and in the Central Region of Romania. The KTC HPI-ULBS must invest further in education, especially since the entrepreneurs placed on the 3rd place in the top of the preferences of technology transfer services the training and assistance services regarding innovation.

Table 3. The main actions of KTC HPI-ULBS for boosting innovation.

\begin{tabular}{l|c}
\hline Actions developed in 2019-2020 at ULBS with the support of HPI Potsdam & Number \\
\hline Developing ULBS Design Thinking school and starting first activities. & 1 \\
Connecting with Global Design Thinking Alliance ${ }^{13}$ managed by HPI Potsdam. & 1 \\
Organizing conferences to increase the visibility of KTC HPI-ULBS: SID & 14 \\
Hackathons / Innovation Labs & 3 \\
Meetings with companies: setting further collaboration activities & 50 \\
Scientific papers based on KTC HPI-ULBS activities & 4 \\
Patent applications & 6 \\
Providing services for/collaborative projects with companies & 21 \\
Investments in R\&D grants for building capacities & 6 \\
Investments in infrastructure of existing research centers/create new ones & 21 \\
Providing educational and vocational training & 10 \\
Partnerships with Digitalization HUBs and IT professional cluster & 3 \\
\hline
\end{tabular}

\section{Conclusions and Further Work}

For boosting regional innovation in Romania a greater collaborative effort is required from clusters, universities and research institutes to position themselves as transfer vectors of technological progress. A top - down approach for boosting innovation should follow the next steps: 1) Romanian government should launch innovation initiatives fostering startups/students interested in founding a company; 2) if this is not possible or feasible, initiatives should be launched at a regional level - supported by digital innovation hubs and collaborative actions between stakeholders; 3) pilot (prototyping) projects between university and third-parties should be showcased at conferences, workshops, or regional networking meetings to serve as 'role models'; 4) learning offers by universities should be jointly developed together with industrial and/or public partners to combine research findings with approaches from practice.

Although the paper presents the HPI-ULBS collaboration aiming territorial innovation in the Sibiu region, this can be considered as innovation pattern that can be applied and extended to other regions or countries, provided it be determined the characteristics of economic competitiveness, the local stakeholders in innovation, both academically and industrially, from the respective region.

As future directions of development, we will carry on with all specified KTC activities, but especially we evaluate the knowledge transfer process in relation to the

${ }^{13}$ https://gdta.org/about-us/member-institutions/\#1600250243638-b971147b-bfb5

14 The Sibiu Innovation Days 2020 - https://events.ulbsibiu.ro/innovationdays/2020.php 
initially set objectives. Examining the effectiveness of knowledge transfer practices is an ongoing effort that needs to be reviewed as the organization grows.

Acknowledgments. This work was partially developed under the ERASMUS+ KA2 project "The FOF-Designer: Digital Design Skills for Factories of the Future", financing contract no. 2018-2553/001-001, project number 601089-EPP-1-2018-1RO-EPPKA2-KA, web: https://www.digifof.eu/ .

\section{References}

1. Hollanders H., Es-Sadki N., Merkelbach I., Khalilova A., European Innovation Scoreboard 2020, Publications Office of the European Union, 2020, ISBN 978-92-76-21528-8.

2. Eurostat - statistics explained, Government expenditure on education, data extracted in February 2021, https://ec.europa.eu/eurostat/statistics-explained/index.php last accessed on March $1^{\text {st }} 2021$.

3. Commission Proposal for Horizon Europe, Horizon Europe - The Next EU Research \& Innovation Investment Programme (2021-2027), May 2019.

4. Etzkowitz, Henry and Leydesdorff, Loet, The Triple Helix - University-IndustryGovernment Relations: A Laboratory for Knowledge Based Economic Development. EASST Review, Vol. 14, No. 1, pp. 14-19, 1995.

5. Carayannis, E. G., Popescu, D., Sipp, C., \& Stewart, M. (2006). Technological learning for entrepreneurial development (TL4ED) in the knowledge economy (KE): Case studies and lessons learned. Technovation, 26(4), 419-443.

6. Curley, M., Salmelin, B., 2018. Open Innovation 2.0. The New Mode of Digital Innovation for Prosperity and Sustainability. Innovation, Technology, and Knowledge Management. Cham, Switzerland: Springer International Publishing.

7. Alexander A., Martin D.P., Manolchev C, Miller K. (2018) University-industry collaboration: using meta-rules to overcome barriers to knowledge transfer, The Journal of Technology Transfer (2020), https://doi.org/10.1007/S10961-018-9685-1

8. Schmiedgen, J., Rhinow, H., Köppen, E., Meinel, C. (2015). Parts without a Whole? - The Current State of Design Thinking Practice in Organizations (Study Report No. 97) (p. 144). Hasso-Plattner-Institut für Softwaresystemtechnik an der Universität Potsdam.

9. Plattner, H., Meinel, C., Leifer, L. (eds.) (2013). Design Thinking - Building Innovation EcoSystems, Springer-Verlag, Berlin, Heidelberg, New York, p. 108.

10. Meinel C., Jahresbericht 2020, Fachgebiet "Internet - Technologien und Systeme", HassoPlattner-Institut für Digital Engineering gGmbH.

11. Camarinha-Matos, L., \& Afsarmanesh, H. (2010). Classes of collaborative networks. In IT Outsourcing: Concepts, Methodologies, Tools, and Applications (pp. 364-370). IGI Global.

12. Nicolaescu, S. S., Palade, H. C., Kifor, C. V., Florea, A. (2017). Collaborative Platform for Transferring Knowledge from University to Industry-A Bridge Grant Case Study, Proceedings of the 4th IETEC Conference, Hanoi, Vietnam, pp. 475-488.

13. Karagiannis, D., Buchmann, R. A., Boucher, X., Cavalieri, S., Florea, A., Kiritsis, D., \& Lee, M. (2020, November). OMiLAB: a smart innovation environment for digital engineers. In Working Conference on Virtual Enterprises (pp. 273-282). Springer, Cham.

14. Florea, A. (2019). Digital Design Skills for Factories of the Future. In MATEC Web of Conferences (Vol. 290, p. 14002). EDP Sciences.

15. The demand for research, development, innovation and technological transfer services among SMEs in the Center Region, http://regio-adrcentru.ro/, last accessed on April 2021. 